\title{
\begin{tabular}{l|l} 
POLITIQUES \& & Politiques et management public
\end{tabular}

\section{Adopter les pratiques de reporting de développement durable : le cas des universités françaises}

Adopting practices of sustainable development reporting: the case of french universities

Stéphanie Chatelain-Ponroy and Sophie Morin-Delerm

(2) OpenEdition

Journals

Electronic version

URL: http://journals.openedition.org/pmp/4922

ISSN: 2119-4831

Publisher

Institut de Management Public (IDPM)

\section{Printed version}

Date of publication: 15 June 2012

Number of pages: $253-280$

ISBN: 978-2-7430-1470-4

ISSN: 0758-1726

Electronic reference

Stéphanie Chatelain-Ponroy et Sophie Morin-Delerm, « Adopter les pratiques de reporting de développement durable : le cas des universités françaises », Politiques et management public [En ligne], Vol 29/2 | 2012, mis en ligne le 18 octobre 2014, consulté le 21 avril 2019. URL : http:// journals.openedition.org/pmp/4922 


\section{Adopter les pratiques de reporting de développement durable : 06 le cas des universités françaises}

\section{Stéphanie Chatelain-Ponroy* et Sophie Morin-Delerm \\ Enseignants-chercheurs au LIRSA EA4603 \\ Conservatoire National des Arts et Métiers \\ 40, rue des Jeûneurs - 75002 Paris}

\section{Résumé}

Les questions liées à la « responsabilité sociale des Entreprise » (RSE) s'étendent désormais à de nombreuses organisations, y compris publiques, en raison de l'importation de techniques et savoirs managériaux dans la sphère publique mais également sous la pression accrue du principe d'accountability. C'est ainsi que les établissements d'enseignement supérieur sont de plus à plus nombreux à intégrer les principes de développement durable dans leurs programmes d'études et de recherche, mais aussi dans leur fonctionnement et dans leurs actions de sensibilisation, d'évaluation et de reporting.

Etudier l'adoption et la diffusion des pratiques de reporting développement durable des universités françaises, nous conduit à observer l'évolution de la communication de ces organisations publiques singulières selon deux dimensions : I'ampleur et la fidélité du reporting.

(c) 2012 IDMP/Lavoisier SAS. Tous droits réservés

Mots clés : pratique organisationnelle, développement durable, reporting, établissement d'enseignement supérieur, université

\section{Abstract}

Adopting practices of sustainable development reporting: the case of french universities. The issues of «corporate social responsibility» (CSR) now extend to many organizations, including public organizations, due to the import of technical and managerial knowledge in the public sphere but also in the increased pressure of the principle of accountability. Thus, the higher education institutions are becoming more likely to integrate the principles of sustainable development into their curricula and research, but also in their operation and their evaluation and reporting.

*Auteur correspondant : stephanie.chatelain@cnam.fr doi:10.3166/pmp.29.253-280 @ 2012 IDMP/Lavoisier SAS. Tous droits réservés 
Studying the adoption and diffusion of sustainable development report by the French universities lead us to observe the evolution of the communication of these specific public organizations through two dimensions: extensiveness and fidelity of reporting.

(c) 2012 IDMP/Lavoisier SAS. Tous droits réservés

Keywords: organizational practices, sustainable development, reporting, higher education institution, university

\section{Introduction}

Au début des années 1980, d'importantes réformes de l'administration et des organisations publiques ont été initiées dans les pays anglo-saxons (Nouvelle Zélande, États-Unis, Grande-Bretagne Australie et Canada) avant d'être développées dans la plupart des pays nordiques et d'Europe continentale. Porteuses de transformations d'envergure de la sphère publique et de sa gouvernance, elles consacrent également l'importation de savoirs managériaux (Bezes, in Lacasse \& Verrier, 2005) et de techniques et outils de gestion au sein des administrations et des organisations publiques (Pollit, 2003 ; Demeestere \& Orange, 2008 ; Lapsley, 2009).

Le principe d'accountability (« redevabilité ») figure parmi les grands préceptes de cette «nouvelle gestion publique ». Il implique que les administrations et organisations publiques rendent davantage compte de leurs activités et de l'exercice de leurs responsabilités sociétales. Pour ce faire, elles ont recours à des instruments qui leur permettent de donner un contenu visible, systématique et crédible à leurs activités et responsabilités sous la forme de reportings destinés à leurs différentes parties prenantes.

Ces tendances (réformes de la gestion publique et accountability) peuvent s'observer dans des domaines variés. Parmi ceux-ci, nous nous intéressons à un mouvement qui, parti des grandes entreprises, s'étend désormais à de nombreuses organisations, y compris publiques, et que l'on désigne sous le terme de CSR (Corporate Social Responsability) ou RSE (Responsabilité Sociale des Entreprises). La « responsabilité sociale » s'impose en effet à présent à l'ensemble des acteurs socio-économiques et institutionnels (MerlinBrogniart \& Depret, 2009) qui sont incités à mettre en œuvre les principes de développement durable et doivent « répondre de leurs actes devant toutes les parties prenantes » (Capron \& Quairel-Lanoizelée, 2007).

Les établissements d'enseignement supérieur constituent un champ privilégié pour observer l'émergence de telles pratiques organisationnelles. En effet, les réformes récentes qui ont touché ces établissements dans de nombreux pays industrialisés (Bleiklie \& Powell, 2005) ont conduit à un rapprochement du modèle universitaire et du modèle de l'entreprise, ce que certains ont qualifié de « capitalisme académique», et se sont traduites par des préoccupations partagées, particulièrement en matière sociétale ${ }^{1}$. Les établissements d'enseignement supérieur

\footnotetext{
${ }^{1}$ Une autre source de cette évolution est sans doute le mouvement vers «l'État exemplaire » qui renvoie à la fois à l'idée que la puissance publique a une responsabilité particulière dans la prise en compte du développement durable, et au fait qu'elle est tenue de rendre des comptes au titre de l'article 15 de la Déclaration des Droits de l'Homme et du Citoyen : «La société peut demander compte à tout agent public de son administration ».
} 
sont ainsi de plus en plus nombreux à intégrer les principes de développement durable dans leurs programmes d'études et de recherche, mais aussi dans leur fonctionnement et leurs actions de sensibilisation, d'évaluation et de reporting (Lozano, 2010).

L'objectif principal de cet article est d'observer, pour mieux les comprendre et les mesurer, l'adoption et la diffusion d'une pratique organisationnelle relativement récente par (et dans) les établissements français d'enseignement supérieur : le reporting développement durable ${ }^{2}$. L'adoption et la diffusion de cette pratique seront évaluées à travers l'évolution de la communication des universités selon deux dimensions : l'ampleur et la fidélité du reporting. Le référentiel Plan Vert ainsi que le guide des Campus Responsables qui le précède servent de matériaux d'études pour montrer l'évolution de l'inscription de la communication développement durable des universités.

Nous examinons notamment quels critères économiques, sociaux et environnementaux sont renseignés par les universités pionnières pour manifester l'adoption et la diffusion du développement durable dans et à l'extérieur de leur organisation. L'inscription dans le champ institutionnel, qui augmente la coercition, mais également la transmission normative et le mimétisme, peut-elle expliquer l'évolution du reporting ? La communication des pratiques du développement durable par les universités « innovateurs » et « adoptants précoces » (Rogers, 1995) est-elle contingente d'éventuelles congruences techniques, politique et culturelle ?

Au-delà de ce travail, cet article constitue la première étape d'une recherche à l'heure où ce mouvement, initié par des établissements pionniers, est appelé à se généraliser. Lors de cette généralisation, le niveau d'engagement des institutions variera vraisemblablement d'un établissement à l'autre, et les professionnels chargés de ce reporting pourront en déterminer l'intensité (ampleur et fidélité).

L'article est construit comme suit. Après cette introduction, nous précisons dans la première section nos cadres théoriques et leurs apports respectifs et complémentaires à notre question de recherche. La deuxième section présente la méthodologie retenue et les résultats empiriques basés sur une analyse comparative (intergroupe, intragroupe et longitudinale) des reportings développement durable des universités. Une dernière section discute de nos résultats en les mettant en perspective grâce aux cadres théoriques mobilisés et souligne les intérêts, limites et perspectives de cette recherche.

\section{Cadres théoriques}

Notre question de recherche-Comment les établissements français d'enseignement supérieur manifestent-ils l'adoption et la diffusion d'une nouvelle pratique organisationnelle ?nous conduit à mobiliser deux cadres théoriques principaux : la théorie néo-institutionnelle, que nous lierons à la théorie de la légitimité, et la théorie de la diffusion de l'innovation. Cette problématique s’inscrit, de façon plus générale, dans la théorie des parties prenantes.

L'approche dominante en matière de développement durable est en effet la théorie des parties prenantes qui place l'entreprise au cœur d'un ensemble de relations avec de multiples parties

\footnotetext{
${ }^{2}$ Ce travail de recherche s'intéresse au reporting développement durable. Dans ce contexte, lorsque nous évoquons les « pratiques », celles-ci recouvrent la " pratique organisationnelle » du reporting (la pratique du rendre-compte) et non les pratiques de développement durable entendues comme des actions responsables.
} 
prenantes. Celles-ci ne se limitent plus aux actionnaires mais à tout « individu ou groupe d'individus, pouvant affecter la performance de l'entreprise ou pouvant être affecté par la réalisation des objectifs de l'organisation » (Freeman, 1984). L'organisation doit alors avant tout être considérée comme « une constellation d'intérêts coopératifs ou concurrents » (Donaldson et Preston, 1995 ; Moore, 1999). Dans les faits, la prise en compte exhaustive de l'ensemble des intérêts des parties prenantes est rarement de mise. Ainsi, dans le secteur de l'enseignement supérieur, les rapports de développement durable construits autour de cinq axes - stratégie et gouvernance ; politique sociale et ancrage territorial ; gestion environnementale ; enseignement et formation ; activité de recherche-concernent toutes les parties prenantes mais intéressent plus particulièrement, à des degrés divers et en fonction des sujets du moment, une ou/et plusieurs parties prenantes. Sur le thème du reporting développement durable, cette réflexion sur les parties prenantes révèle les intérêts contradictoires et/ou complémentaires exerçant une pression multiforme sur les organisations et renvoie à la question de l'accountability (Cooper \& Owen, 2007), centrale dans le champ du NPM. Cependant, la stakeholder theory - notamment parce qu'elle est avant tout une approche contractualiste - peut paraître insuffisante pour analyser et comprendre le développement des reportings développement durable dans des organisations peu soumises au marché ${ }^{3}$ comme le sont encore les universités françaises. Il est par conséquent nécessaire d'examiner les contraintes qui pèsent sur ces organisations et les conduisent à se conformer aux attentes externes. La théorie néo-institutionnelle et la théorie de la légitimité offrent à cet égard des grilles de lecture et d'analyse fécondes.

\subsection{Les théories néo-institutionnelle et de la légitimité}

La théorie néo-institutionnelle postule que le contexte institutionnel est nécessaire pour comprendre le fonctionnement des organisations. Ces dernières sont ainsi structurées par leur environnement : elles deviennent isomorphes à ce dernier en incorporant en leur sein des éléments leur permettant de gagner en légitimité dans leur champ organisationnel, et ce indépendamment de considérations rationnelles ou d'efficience. Ce sont donc des entités sociales, encastrées dans des réseaux complexes de croyances, règles et rôles institutionnalisés (Meyer \& Rowan, 1977), de schémas culturels et de conventions qui façonnent leurs buts, formes et pratiques organisationnelles dans un mouvement d'homogénéisation lié au phénomène d'isomorphisme institutionnel (DiMaggio \& Powell, 1983). Ce mouvement est lié aux pressions institutionnelles qui pèsent sur les organisations et vont les conduire à converger sous l'influence d'isomorphismes coercitif, normatif et mimétique.

Même si, sur le plan empirique, ces trois phénomènes sont difficiles à distinguer, le développement de rapports de développement durable au sein des universités peut être lu comme une homogénéisation des pratiques, élaborées et reproduites au sein du champ (Scott, 2001 ; Collier, 2001), traduisant une forme d'isomorphisme institutionnel et motivées par une recherche de légitimité. Cette homogénéisation agirait alors comme « signal » de conformité aux systèmes de croyances et de règles du champ et permettrait, de ce fait, un accès facilité aux ressources.

\footnotetext{
${ }^{3}$ Même si l'on peut légitimement penser qu'une université ait des raisons propres, au titre de sa vocation pédagogique, de s'engager dans le développement durable et la RSE et que l'imitation de l'entreprise ne soit pas le seul moteur...
} 
Cette quête - centrale dans la théorie institutionnelle - de légitimité au sein de leur environnement institutionnel explique qu'un pont nous semble devoir être lancé en direction de la théorie de la légitimité. En effet, la légitimité, entendue comme « hypothèse ou perception généralisée que les actions d'une entité sont désirables, propres et appropriées à l'intérieur d'un système de normes, de valeurs, de croyances et de définitions socialement construites » (Suchman, 1995) est le moteur principal de l'isomorphisme institutionnel. Elle assure à l'organisation l'approbation de la société et lui donne la possibilité d'obtenir les ressources dont elle a besoin pour survivre : «l'acceptabilité sociale qui résulte de la légitimité peut être plus importante que la performance économique » (Pfeffer \& Salancik, 1978). À ce titre, la légitimité organisationnelle devient une ressource stratégique dont dépend la survie de l'organisation (Déjean \& Oxibar, 2005) liée, en partie, aux réponses apportées aux attentes des parties prenantes et notamment au respect d'un contrat tacite imposant à l'entreprise une obligation informelle de contribuer à l'augmentation du bien-être de la société, afin de pouvoir exercer son activité sans entrave et d'être acceptée par la collectivité sans tomber dans le discrédit.

L'apport de la théorie de la légitimité permet de mieux comprendre l'engagement des universités, toujours plus nombreuses, dans une démarche de développement durable. La légitimité est déterminée, entre autres, par le domaine d'activité, le mode de production, les produits, les buts... de l'organisation et « [...] ne pouvant plus être établie a priori, elle devient un problème managérial et une préoccupation majeure pour le dirigeant, [...] il lui faut montrer, par des exemples concrets, que son activité peut-être bénéfique à la communauté politique et sociale qui l'héberge. » (Laufer, 1996). Le développement durable oblige ainsi l'université à repenser sa communication et ses actes pour informer ses parties prenantes qu'elle ne se limite pas à la seule transmission du savoir mais exerce un rôle social en participant aux évolutions économiques, écologiques et sociales de la société. En la matière, la variété des parties prenantes rend la communication encore plus délicate. On peut ainsi s'interroger sur la place accordée à la légitimité aux yeux des acteurs internes (le reporting développement durable est-il légitime pour les directeurs de composantes, pour les directeurs de laboratoires, pour les enseignants chercheurs ?). Pourquoi, lorsqu'une organisation parle de développement durable, fait-elle l'objet d'une suspicion légitime? Sans doute parce qu'elle entre dans un cercle restreint de spécialistes, côtoie des ONG, etc. La quête de légitimité du développement durable est générée par ce doute originel. Un comportement éthique, des discours et des actions concrètes de développement durable insufflés dans tout l'établissement, constituent des instruments privilégiés de gestion de la légitimité, de compétences distinctives appréciées des parties prenantes, et donc, on peut l'imaginer, de performance (Castelnau \& Noël, 2004 ; Capron \& Quairel- Lanoizelée, 2004).

\subsection{La théorie de la diffusion de l'innovation}

Enfin, parce qu'ils conduisent les universités à adopter de nouvelles normes, l'introduction et le développement de reportings développement durable dans les universités peuvent s'apparenter à l'intégration d'une innovation et à sa diffusion. Un deuxième cadre théorique peut, par conséquent, être mobilisé pour comprendre comment est adoptée et diffusée cette nouvelle pratique.

Selon Rogers (1995), pour qu'une nouvelle idée soit adoptée, il faut que les organisations en perçoivent l'avantage : que cette « innovation » soit compatible avec leurs valeurs, facile à adopter - qu'elle n'implique pas de changements trop radicaux de l'organisation -, que les 
organisations puissent la mettre en place progressivement à coup d'essais-erreurs et enfin que les résultats soient visibles. En outre, l'innovation-décision mise en œuvre par les membres d'un système social dépend aussi fortement de l'innovation-décision des autres membres de ce système (Rogers, 1962, 1995; Zaltman, Duncan, \& Holbeck, 1973). La diffusion du développement durable dans les établissements d'enseignement supérieur est ainsi encouragée par l'adoption de nouvelles règles et lois, par la mise à disposition d'outils (référentiels, systèmes d'auto-évaluation, guides de bonnes pratiques, ...) et par une demande de plus en plus prégnante des parties prenantes des universités. De surcroît, les universités rétives et retardataires - supportant les injonctions d'autres institutions engagées à rendre des comptes à travers leur rapport d'activité, auprès d'organismes de notation ou d'accréditation - se voient dans l'obligation de communiquer sur le thème du développement durable. Nous assistons ici à une diffusion verticale descendante sous contrainte qui a tendance à faire accélérer la communication de l'adoption du développement durable dans l'ensemble des universités. Ainsi, alors qu'en juillet 2007, seulement huit universités françaises avaient choisi de participer au guide «Campus Responsable » et de montrer par cette action de communication qu'elles adoptaient les principes du développement durable, en novembre 2010 vingt universités étaient dénombrées. D'un point de vue plus global, la diffusion du développement durable, de sa communication et de ses pratiques, d'abord par des établissements visibles et reconnus ${ }^{4}$, qui sont peu à peu ralliés par les autres, peut être analysée à travers ce prisme.

Rogers identifie trois types d'innovation-décision qui fondent la diffusion : l'innovationdécision optionnelle - prise par un individu singulier -, collective - prise de façon consensuelle par tous les individus d'un système social -, et autoritaire - prise pour le système social dans son entier par quelques individus, souvent leaders charismatiques. Dans le cas de la diffusion des rapports de développement durable au sein des universités françaises, l'innovation-décision semble être, dans un premier temps, plutôt de type optionnel puisque le développement durable a d'abord été adopté par quelques rares établissements ; puis, dans un second temps, de type à la fois autoritaire (au sens de Rogers, 1995) - avec la mise en place de dispositifs législatifs exécutés par les chefs d'établissements - et collectif car de multiples établissements s'engagent de plus en plus spontanément en faveur de cette cause.

Chacun de ces deux cadres théoriques comporte, nous l'avons vu, des limites mais nous éclaire aussi sur les phénomènes étudiés. Nous pensons donc que leur combinaison nous permet de mieux comprendre l'émergence récente de rapports développement durable dans les universités françaises en nous conduisant à identifier des « catégories » d'innovateurs et à montrer comment ceux-ci répondent de manière différenciée aux injonctions de leur champ institutionnel. Pour cela, à l'instar d'Ansari \& al. (2010), nous avons choisi de nous intéresser à l'inscription des pratiques organisationnelles - relatives au reporting développement durable - et à leur diffusion (dans le temps et entre les organisations) en montrant comment le processus de diffusion résulte d'un ajustement dynamique - rationnel et social - entre la pratique et l'organisation qui l'intègre, et en soulignant combien la forme particulière adoptée est influencée par des facteurs techniques, culturels et politiques. Il s'agira alors d'évaluer le niveau de congruence technique (compatibilité technique de la nouvelle pratique orga-

\footnotetext{
${ }^{4}$ Les premiers adhérents à la campagne 2006 des campus responsables (qui a donné naissance au $1^{\mathrm{er}}$ guide des campus responsables en 2007) étaient principalement des écoles (Bordeaux EM, Euromed Marseille, HEC, UTT, Escem, ESCP-EAP, HEMA, ESC Rouen, ESC Brest, ESC Reims).
} 
nisationnelle avec les technologies mobilisées par les organisations adoptantes), culturelle (compatibilité de la nouvelle pratique organisationnelle avec les valeurs, croyances et pratiques mobilisées par les organisations adoptantes) et politique (compatibilité des caractéristiques normatives de la nouvelle pratique organisationnelle avec les intérêts et priorités (agendas) des organisations adoptantes) pour expliquer le degré d'adoption de l'innovation.

En outre, les pratiques organisationnelles elles-mêmes peuvent présenter des caractéristiques intrinsèques qui rendent plus ou moins aisée leur intégration par les organisations adoptantes. Ansari \& al. (2010) listent ainsi trois caractéristiques : l'interprétativité des pratiques, leur divisibilité et leur complexité.

L' « interprétativité » des pratiques indique la possibilité d'adaptation de certaines pratiques qui se prêtent parfois à de multiples interprétations. Les pratiques qui résultent de dispositions juridiques sont ainsi moins susceptibles d'être adaptées en fonction de leur sens, alors que celles qui sont définies de façon moins stricte offrent davantage de possibilités d'interprétation divergentes (Benders \& van Veen, 2001). La « divisibilité » des pratiques souligne la possibilité d'adoption des pratiques à une petite échelle. Cette notion se rapproche de celle d'essayabilité, qui est « la mesure selon laquelle une innovation peut être expérimentée sur une base limitée » (Rogers, 1995, p.243). Enfin, la « complexité » pointe la difficulté à comprendre et à mettre en œuvre des pratiques organisationnelles en raison notamment de leur ambiguïté liée au grand nombre d'interfaces sociales qu'elles nécessitent. Cette notion se rapproche de celle de « complexité » de Rogers (1995).

Ces trois caractéristiques qui conditionnent la facilité - ou non - de l'intégration des pratiques organisationnelles constituent une aide à l'interprétation de l'inscription des pratiques ainsi que de leur évolution. Elles nous permettront de mieux comprendre l'adoption et la diffusion des pratiques de reporting.

Deux dimensions sont alors proposées par Ansari \& al. (2010) pour qualifier les variations observées des pratiques organisationnelles : la fidélité, entendue comme la ressemblance plus ou moins grande de la nature de la pratique adoptée avec une version précédente de référence ou un prototype, et l'ampleur, qui évalue le degré de mise en œuvre de la pratique par rapport à celui de la version de référence.

Figure 1 : Les dimensions des variations de pratiques organisationnelles (adaptée de Ansari \&al. 2010)

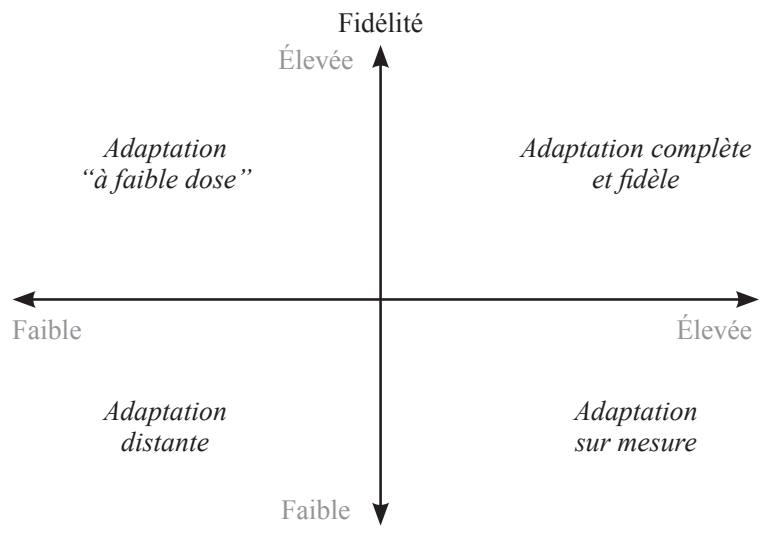


À partir de ce cadre, des liens peuvent être dressés entre les caractéristiques des pratiques organisationnelles et leurs variations observées. De plus, des trajets dynamiques des pratiques, directement contingents de facteurs techniques, culturels et politiques, peuvent être esquissés. L'intégration du reporting développement durable par les universités françaises pourrait être observée selon ce prisme.

Ainsi par exemple, Ansari et al. énoncent qu'une plus grande «interprétativité » encouragerait une adaptation moins fidèle, mais n'aurait pas d'incidence sur l'ampleur de sa mise en œuvre. De même, une grande divisibilité permettrait une adaptation réduite dans son ampleur mais n'affecterait pas la fidélité d'une pratique. Enfin, une complexité accrue de la pratique entraînerait une adaptation fortement fidèle mais peu vaste. Du point de vue du processus de diffusion des pratiques de reporting, les variations de congruence technique, culturelle et/ou politique conditionneraient les niveaux d'ampleur et de fidélité et par conséquent l'évolution des pratiques de reporting.

Après avoir présenté les cadres théoriques qui constituent le socle de notre travail et mobilisé la réflexion multi-facettes d'Ansari \& al. (2010) qui proposent un prisme d'analyse des pratiques organisationnelles riche et englobant des éléments essentiels constitutifs de nos cadres, nous pouvons entamer la section II. Elle présente la méthodologie retenue et les résultats empiriques basés sur une analyse comparative (intergroupe, intragroupe et longitudinale) des reportings développement durable des universités.

\section{Méthodologie et résultats empiriques}

Au cours de cette section, nous présenterons donc tout d'abord le champ institutionnel avec la stratégie nationale de développement durable, le Grenelle de l'Environnement et le Plan Vert. Puis nous décrirons les thèmes du Guide Campus Responsable, document précédant le référentiel Plan Vert et servant de trame aux premiers reportings. Enfin, nous présenterons le recueil des données empiriques.

\subsection{Le champ institutionnel}

Au sein du champ institutionnel de l'enseignement supérieur coexistent différents jeux de pressions coercitives et normatives qui se sont intensifiés récemment et poussent les établissements français vers une homogénéisation isomorphique : textes réglementaires, référentiels normatifs, standards d'évaluation, organismes certificateurs porteurs d'une logique de normalisation du développement durable... Nous nous concentrerons ici sur trois des pressions récentes les plus spécifiques aux établissements d'enseignement supérieur : Stratégie nationale du développement durable, Grenelle Environnement et Plan Vert (encadré 1). Nous n'oublions pas cependant que ces établissements, à l'instar de toutes les organisations des pays industrialisés, sont soumis à une pression globale en même temps que diffuse sur le thème du développement durable. Celle-ci se traduit par le fait que la communication vis-à-vis des parties prenantes en est devenue incontournable (GRI, Climate change act 2008, Sustainabiliy Reporting Toolkit, Agenda 21...). Les entreprises cotées sur un marché règlementé, par exemple, doivent présenter leur démarche développement durable dans leurs rapports annuels, et toute société de plus de 500 salariés, cotée ou non, y est très fortement encouragée. 


\section{Encadré 1 : Trois dispositifs porteurs de pressions institutionnelles}

La stratégie nationale du développement durable, mise en place dès 2003 et renouvelée en 2010, offre un cadre de référence et d'orientation pour l'ensemble des acteurs privés et publics, en cohérence avec la stratégie des instances européennes et avec les engagements internationaux de la France. Elle rend nécessaire l'intégration du développement durable dans les modalités de fonctionnement interne des services de l'État et fixe à ces derniers des objectifs quantifiés d'éco-responsabilité dans les domaines de l'énergie, de l'eau, des déchets, des achats, des bâtiments, des transports, des gaz à effets de serre. Des indicateurs de suivis sont mis en place et restitués, notamment par les rapports d'activités ministériels et un rapport annuel. En leur qualité d'institutions publiques, les établissements d'enseignement supérieur sont concernés par l'ensemble des défis de la stratégie nationale du développement durable.

Le Grenelle Environnement, initié en 2007, est un ensemble de rencontres politiques réunissant l'État et les représentants de la société civile afin de définir une feuille de route en faveur de l'écologie, du développement et de l'aménagement durables. Avec les lois « Grenelle 1 », « Grenelle 2 » et « Grenelle 3 », le Parlement a adopté les textes nécessaires à la traduction législative des engagements du Grenelle Environnement. Les établissements d'enseignement supérieur sont plus directement concernés par les domaines d'action «Recherche » et «Etat exemplaire », et l'article 55 du Grenelle 1 qui leur est consacré stipule qu'ils « élaboreront, pour la rentrée 2009, un "Plan Vert” pour les campus. Les universités et grandes écoles pourront solliciter une labellisation sur le fondement de critères de Développement durable ».

Le Plan Vert constitue par conséquent une obligation législative (pression coercitive) qui s'impose aux établissements.

Un Plan Vert a donc été élaboré par la Conférence des Présidents d'Université (CPU) et la Conférence des Grandes Écoles (CGE). Le Plan Vert est un «plan développement durable » qui recouvre toutes les dimensions du développement durable et vise à sa bonne intégration par les établissements d'enseignement supérieur dans leurs missions pédagogiques et de recherche, mais aussi dans leur gouvernance. Il constitue un référentiel « Développement durable » pour les établissements d'enseignement supérieur qui sont invités à l'utiliser pour mettre en place des actions en faveur du développement durable et solliciter une labellisation. Chaque établissement est laissé libre de mettre en œuvre à son rythme et en fonction de sa situation propre le modèle de stratégie de développement durable qui lui correspond (canevas de Plan Vert) et l'outil de pilotage de cette stratégie (référentiel « développement durable»). Ce référentiel constitue, en outre, un outil de communication et de dialogue avec les parties prenantes mais aussi un instrument de partage de bonnes pratiques. Il est, en effet, un cadre commun à tous les établissements qui doit permettre un recensement et une diffusion des bonnes pratiques pour favoriser un large déploiement des Plans Verts dans l'enseignement supérieur. En ce sens, il exerce une pression non seulement normative mais également mimétique sur les établissements. Enfin, il s'inscrit dans une démarche de labellisation dont il constitue la première étape. Un outil d'auto-évaluation (Evaddes) automatise et facilite l'utilisation pratique du référentiel en permettant à chaque établissement de réaliser son auto-évaluation, de suivre l'avancement de son Plan Vert, d'évaluer et de réviser son plan d'actions, de rédiger et publier son rapport. 
Ces trois dispositifs - stratégie nationale de développement durable, Grenelle Environnement, Plan Vert - exercent sur les établissements d'enseignement supérieur des pressions institutionnelles qui les poussent à homogénéiser leurs pratiques en matière de rapport de développement durable. Ces dispositifs, qui se succèdent et s'imbriquent, permettent en effet de comprendre comment les pressions coercitives, normatives et mimétiques se succèdent, se complètent et s'additionnent. Schématiquement on peut observer une déclinaison des préceptes généraux vers des indicateurs détaillés, des énoncés politiques vers des traductions réglementaires puis opérationnelles, d'une vision interorganisationnelle vers un prisme intra-organisationnel.

Tableau 1 : Gradation des pressions isomorphiques

\begin{tabular}{|l|c|c|c|}
\hline Isomorphisme & $\begin{array}{c}\text { Stratégie nationale du } \\
\text { développement durable }\end{array}$ & Grenelle Environnement & Plan Vert \\
\hline Coercitif & $\mathbf{x}$ & $\mathbf{x}$ & $\mathbf{x}$ \\
\hline Normatif & & $\mathbf{x}$ & $\mathbf{x}$ \\
\hline Mimétique & & & $\mathbf{x}$ \\
\hline
\end{tabular}

\subsection{Les établissements d'enseignement supérieur}

Jusqu'en 2006, et alors même que de nombreux programmes de formation existaient déjà sur ce thème, très peu d'établissements d'enseignement supérieur français avaient entamé une démarche globale de développement durable. Pour encourager celle-ci, une initiative baptisée «Campus Responsable » a été créée en 2005 pour favoriser une émulation entre les campus et distinguer les établissements pionniers en rendant visibles leurs pratiques en faveur de la promotion du développement durable. Cette initiative a fondé une campagne baptisée «Campus Responsable » en juin 2006 à laquelle les établissements d'enseignement supérieur ont pu adhérer dès mars 2007.

La première édition du Guide des Campus Responsables date de 2007. Elle est fondée sur un questionnaire d'auto-évaluation adressé à 282 universités et grandes écoles et a pour objectif de dresser un panorama des bonnes pratiques menées sur les campus français, de signaler les meilleurs campus sur des thèmes donnés mais aussi d'informer les étudiants et le grand public sur les projets menés par les campus dans le domaine du développement durable. Alors que 22 établissements seulement (dont 8 universités) avaient répondu lors de la première édition ce guide, ils étaient 58 (dont 20 universités) à participer à l'édition 2010.

Cet accroissement peut être lu comme une prise de conscience plus forte de la part des établissements de la nécessité de communiquer sur le thème du développement durable mais aussi comme le fruit des pressions plus importantes qui pèsent sur les établissements. En effet, entre la première et la dernière version du guide, a été mise en place le dispositif «Plan Vert » et son référentiel, né de la fusion du référentiel Campus Responsables avec celui de la Conférence des Présidents d'Université (CPU). Le référentiel est donc à présent commun à tous les établissements d'enseignement supérieur en France. 
Cinq grandes thématiques structurent les différentes éditions des guides des Campus Responsables : stratégie et gouvernance, politique sociale et ancrage territorial, gestion environnementale, enseignement et formation et activités de recherche. Le référentiel offre la possibilité aux campus de s'auto-évaluer sur leur niveau d'avancement en s'attribuant une note de 1 à 4 sur la centaine de questions qui le compose, ce qui permet de les doter d'une note moyenne par thématique et ainsi de les positionner sur les cinq thématiques.

La thématique «Stratégie et gouvernance » rend compte de l'engagement de la présidence ou direction de l'établissement au travers de sa politique générale et de ses relations avec les différentes parties prenantes, qu'il s'agisse de les sensibiliser, de les consulter ou encore de les impliquer sur les décisions les concernant. Cette thématique est composée de quatre dimensions : Engagement de la présidence ; Prospective, planification et réalisation ; Gouvernance participative et Efficacité budgétaire.

La thématique « Politique sociale et ancrage territorial » indique les politiques sociales mises en place ou soutenues par l'établissement en faveur du personnel et des étudiants. Elle comprend également la participation du campus à des projets locaux et internationaux. Cette thématique est composée de quatre dimensions : Équité sociale ; Développement individuel ; Ancrage territorial et Efficacité budgétaire.

La thématique « Gestion environnementale » s'intéresse à la prise en compte des impacts environnementaux directs du campus : politique de construction ou rénovation durable des bâtiments, politiques et performances en matière d'éco-efficacité, intégration de critères environnementaux dans les achats, offre de restauration responsable, protection de la biodiversité, plan de déplacement... Cette thématique est composée de quatre dimensions : Stratégie de l'établissement ; Gestion des ressources et intrants ; Gestion des extrants et Gestion de la qualité de l'environnement.

La thématique «Enseignement et formation » porte sur l'intégration des enjeux du développement durable dans la formation initiale et continue des étudiants. Elle indique les cours, stages et projets pour les étudiants, les outils pédagogiques et formation pour les enseignants.

La thématique «Activité de recherche » concerne l'orientation de la recherche sur les thèmes relatifs au développement durable mais aussi les missions de conseil, partenariats privés, structures de veille et travaux publiés...

Nous nous limitons, pour cette étude exploratoire, à l'analyse des trois premières thématiques. La formation et la recherche, parce qu'elles constituent l'activité même des établissements, sont en effet des axes spécifiques aux universités et leur analyse ne permettrait vraisemblablement pas de manifester de singularités organisationnelles.

\subsection{Le recueil des éléments empiriques}

Selon le modèle préconisé par Miles \& Huberman (1994) nous avons successivement condensé les données, organisé et présenté celles-ci, puis nous en proposons une interprétation.

La condensation et le codage des données de reporting nous ont permis de transformer la multitude de ces dernières en un ensemble organisé nous autorisant à progresser dans notre question de recherche. Les matrices de présentation ont été réalisées à partir de nos cadres théoriques et des données recueillies afin de mettre en valeur les liens entre nos observations et d'en proposer des interprétations. 


\section{Encadré 2 : Choix des établissements étudiés}

Les 58 établissements participant au Guide des Campus Responsables 2010 sont constitués de 20 universités, 22 écoles de commerce, 3 établissements spécialisés et 13 écoles d'ingénieurs. Nous avons tout d'abord réalisé une première recherche d'archives sur l'ensemble de ces établissements français pour examiner les stratégies multiples qu'ils mettaient en œuvre afin de communiquer sur leurs pratiques de développement durable. Cependant, la variété des établissements - et notamment le fait que les parties prenantes ne sont pas les mêmes pour les universités et les grandes écoles, que la coercition s'y exerce de façon différente, que la recherche de légitimité n'y est pas identique - nous a conduites à réaliser une étude exploratoire plus approfondie sur certains d'entre eux afin de mieux observer les évolutions. Les cas particuliers étudiés ont alors été sélectionnés pour illustrer les processus d'institutionnalisation et de déclinaison des pratiques organisationnelles au sein du champ des établissements d'enseignement supérieur.

Cette focalisation nous a amenées à formuler nos questionnements empiriques comme suit : Quels sont les établissements universitaires « innovateurs», premiers entrants présents dans l'édition $n^{\circ} 1$ du guide Campus Responsables (4 juillet 2007) et qui y sont encore dans la troisième édition (8 novembre 2010) ? Peut-on mesurer une évolution de la fidélité et de l'ampleur de leurs pratiques témoignant de leur engagement en faveur du développement durable?

En outre, les établissements qui les suivent immédiatement, que l'on peut qualifier d'« adoptants précoces », sont également considérés et l'évolution de leurs pratiques étudiée. Existe-t-il, d'une part, une différence entre ces deux groupes d'établissements en termes d'intensité de la fidélité et de l'ampleur au moment de l'entrée dans le processus et, d'autre part, une évolution de cette intensité en termes d'ampleur et de fidélité lors de l'ancrage dans le processus?

En trois ans et trois mois, sur huit institutions universitaires initialement adhérentes, deux n'ont pas fourni de données de suivi (Troyes, Grenoble et Franche Comté) et cinq restent adhérentes et actives dans la troisième édition du guide. Nous les qualifions d'établissements « innovateurs » au sens de Rogers (1995) car impliqués sur le thème du développement durable les premiers et bien avant les pressions réglementaires. Ces cinq innovateurs sont suivis par cinq autres institutions universitaires dont une seule (Albi) n'a pas fourni de données de suivi. Les quatre restantes sont donc étudiées en leur qualité d' «adoptants précoces ». 
Les établissements observés ont fait l'objet d'un choix (encadré 1) et la liste de ceux qui ont été finalement retenus figure dans le tableau 2.

Tableau 2 : Les établissements étudiés

\begin{tabular}{|c|c|}
\hline $\begin{array}{c}\text { Établissements appartenant à la catégorie } \\
\text { des innovateurs }\end{array}$ & $\begin{array}{c}\text { Établissements appartenant à la catégorie } \\
\text { des adoptants précoces }\end{array}$ \\
\hline $\begin{array}{c}\text { Observatoire de Paris } \\
\text { Université de Caen } \\
\text { Université Nantes } \\
\text { Université du Havre }\end{array}$ & $\begin{array}{c}\text { Université d'Angers } \\
\text { Université de Bretagne Occidentale (UBO) } \\
\text { Université de Sud Toulon-Var } \\
\text { Université René Cassin Lyon (UPI) }\end{array}$ \\
\hline Université de Saint-Étienne (Jean Monnet) & \begin{tabular}{c} 
\\
\hline
\end{tabular}
\end{tabular}

Pour percevoir l'évolution des pratiques de reporting en matière de développement durable nous les avons analysées selon deux prismes :

- Un premier prisme temporel, nous conduit à distinguer la période d'entrée dans le processus - celles qui correspondent à l'année au cours de laquelle l'établissement rejoint l'initiative Campus Responsable et fournit pour la première fois ses données - de celle d'ancrage dans le processus. Pour cette dernière, nous avons choisi d'observer les dernières informations dont nous disposions (2010) afin de mieux visualiser d'éventuelles évolutions entre les deux périodes.

- Un second prisme vise à qualifier la nature des pratiques de reporting au travers des deux dimensions mises en évidence par Ansari (Ansari \& al. 2010) : l'ampleur, qui indique le degré d'intensité des pratiques, est appréciée par la note moyenne attribuée par l'organisation «Campus Responsable » à partir des éléments communiqués par les établissements puisque celle-ci évalue le degré de mise en œuvre de la pratique. Comme la fidélité, elle est propre à chaque thématique. La fidélité des pratiques est, quant à elle, appréciée par l'existence d'un reporting sur chacune des dimensions constitutives des cinq thématiques associées au développement durable (version de référence). Autrement dit, si un établissement ne mentionne, dans son reporting développement durable, d'éléments que pour l'une des x dimensions constitutives de l'une des cinq thématiques, il obtient une note de fidélité de 1/x pour cette thématique ; s'il n'indique, dans son reporting développement durable, d'éléments que pour deux des x dimensions constitutives de l'une des cinq thématiques, il obtient une note de fidélité de $2 / x$ pour cette thématique, etc.

Pour chaque établissement et chaque période une grille d'analyse nous a permis de synthétiser les éléments collectés et de les rendre comparables d'un établissement à l'autre. Nous donnons en annexe comme exemple la grille de synthèse de l'université de Nantes. Les éléments de synthèse des neuf établissements sont indiqués dans les tableaux 3 et 4 . 
Tableau 3 : Fidélité et ampleur des pratiques de reporting développement durable des innovateurs (synthèse)

\begin{tabular}{|c|c|c|c|c|c|c|}
\hline 1. Université de Caen & \multicolumn{2}{|c|}{$\begin{array}{c}\mathrm{P} 1- \\
\text { Entrée dans } \\
\text { le processus }\end{array}$} & \multicolumn{2}{|c|}{$\begin{array}{c}\mathrm{P} 2- \\
\text { Ancrage dans } \\
\text { le processus }\end{array}$} & \multicolumn{2}{|c|}{ Évolution } \\
\hline Axes & Ampleur & Fidélité & Ampleur & Fidélité & Ampleur & Fidélité \\
\hline Stratégie et gouvernance & 2 & 2 & 2 & 3 & -- & +1 \\
\hline $\begin{array}{l}\text { Politique sociale et } \\
\text { ancrage territorial }\end{array}$ & 2 & 1 & 2 & 2 & -- & +1 \\
\hline Gestion environnementale & 4 & 3 & 1 & 4 & -3 & +1 \\
\hline $\begin{array}{l}\text { Total ampleur / total } \\
\text { fidélité }\end{array}$ & 8 & 6 & 5 & 9 & -3 & +3 \\
\hline $\begin{array}{l}\text { 2. Observatoire } \\
\text { astronomique de Paris }\end{array}$ & \multicolumn{2}{|c|}{$\begin{array}{l}\mathrm{P} 1- \\
\text { Entrée dans le } \\
\text { processus }\end{array}$} & \multicolumn{2}{|c|}{$\begin{array}{c}\mathrm{P} 2- \\
\begin{array}{c}\text { Ancrage dans le } \\
\text { processus }\end{array}\end{array}$} & \multicolumn{2}{|c|}{ Évolution } \\
\hline Axes & Ampleur & Fidélité & Ampleur & Fidélité & Ampleur & Fidélité \\
\hline Stratégie et gouvernance & 1 & 2 & 2 & 2 & +1 & -- \\
\hline $\begin{array}{l}\text { Politique sociale et } \\
\text { ancrage territorial }\end{array}$ & 1 & 1 & 2 & 2 & +1 & +1 \\
\hline Gestion environnementale & 4 & 4 & 2 & 4 & -2 & -- \\
\hline $\begin{array}{l}\text { Total ampleur / total } \\
\text { fidélité }\end{array}$ & 6 & 7 & 6 & 8 & -- & +1 \\
\hline 3. Université de Nantes & \multicolumn{2}{|c|}{$\begin{array}{c}\mathrm{P} 1- \\
\text { Entrée dans le } \\
\text { processus }\end{array}$} & \multicolumn{2}{|c|}{$\begin{array}{c}\mathrm{P} 2- \\
\text { Ancrage dans le } \\
\text { processus }\end{array}$} & \multicolumn{2}{|c|}{ Évolution } \\
\hline Axes & Ampleur & Fidélité & Ampleur & Fidélité & Ampleur & Fidélité \\
\hline Stratégie et gouvernance & 4 & 3 & 3 & 2 & -1 & -1 \\
\hline $\begin{array}{l}\text { Politique sociale et } \\
\text { ancrage territorial }\end{array}$ & 2 & 3 & 2 & 2 & -- & -1 \\
\hline Gestion environnementale & 3 & 4 & 2 & 3 & -1 & -1 \\
\hline $\begin{array}{l}\text { Total ampleur / total } \\
\text { fidélité }\end{array}$ & 9 & 10 & 7 & 7 & -2 & -3 \\
\hline 4. Université du Havre & \multicolumn{2}{|c|}{$\begin{array}{c}\mathrm{P} 1- \\
\text { Entrée dans le } \\
\text { processus }\end{array}$} & \multicolumn{2}{|c|}{$\begin{array}{c}\mathrm{P} 2- \\
\text { Ancrage dans le } \\
\text { processus }\end{array}$} & \multicolumn{2}{|c|}{ Évolution } \\
\hline Axes & Ampleur & Fidélité & Ampleur & Fidélité & Ampleur & Fidélité \\
\hline Stratégie et gouvernance & 4 & 3 & 3 & 2 & -1 & -1 \\
\hline $\begin{array}{l}\text { Politique sociale et } \\
\text { ancrage territorial }\end{array}$ & 3 & 2 & 3 & 3 & -- & +1 \\
\hline Gestion environnementale & 4 & 4 & 3 & 4 & -1 & -- \\
\hline $\begin{array}{l}\text { Total ampleur / total } \\
\text { fidélité }\end{array}$ & 11 & 9 & 9 & 9 & -2 & - \\
\hline
\end{tabular}




\begin{tabular}{|c|c|c|c|c|c|c|}
\hline $\begin{array}{l}\text { 5. Université Jean } \\
\text { Monnet } \\
\text { de Saint-Étienne }\end{array}$ & \multicolumn{2}{|c|}{$\begin{array}{c}\mathrm{P} 1- \\
\text { Entrée dans le } \\
\text { processus }\end{array}$} & \multicolumn{2}{|c|}{$\begin{array}{c}\mathrm{P} 2- \\
\text { ancrage dans le } \\
\text { processus }\end{array}$} & \multicolumn{2}{|c|}{ Évolution } \\
\hline Axes & Ampleur & Fidélité & Ampleur & Fidélité & Ampleur & Fidélité \\
\hline Stratégie et gouvernance & 1 & 1 & 2 & 3 & +1 & +2 \\
\hline $\begin{array}{l}\text { Politique sociale et } \\
\text { ancrage territorial }\end{array}$ & 1 & 1 & 3 & 1 & +2 & -- \\
\hline Gestion environnementale & 1 & 1 & 2 & 3 & +1 & +2 \\
\hline $\begin{array}{l}\text { Total ampleur / } \\
\text { total fidélité }\end{array}$ & 3 & 3 & 7 & 7 & +4 & +4 \\
\hline
\end{tabular}

Tableau 4 : Fidélité et ampleur des pratiques de reporting développement durable des adoptants précoces (synthèse)

\begin{tabular}{|c|c|c|c|c|c|c|}
\hline $\begin{array}{l}\text { 6. Université de } \\
\text { Bretagne occidentale }\end{array}$ & \multicolumn{2}{|c|}{$\begin{array}{c}\mathrm{P} 1- \\
\text { Entrée dans le } \\
\text { processus }\end{array}$} & \multicolumn{2}{|c|}{$\begin{array}{c}\mathrm{P} 2- \\
\text { Ancrage dans le } \\
\text { processus }\end{array}$} & \multicolumn{2}{|c|}{ Évolution } \\
\hline Axes & Ampleur & Fidélité & Ampleur & Fidélité & Ampleur & Fidélité \\
\hline Stratégie et gouvernance & 2 & 2 & 2 & 2 & -- & -- \\
\hline $\begin{array}{l}\text { Politique sociale et ancrage } \\
\text { territorial }\end{array}$ & 1,5 & 2 & 3 & 3 & $+1,5$ & +1 \\
\hline Gestion environnementale & 3 & 2 & 2 & 2 & -1 & -- \\
\hline $\begin{array}{l}\text { Total ampleur / } \\
\text { total fidélité }\end{array}$ & 6,5 & 6 & 7 & 7 & $+0,5$ & +1 \\
\hline 7. Université d'Angers & \multicolumn{2}{|c|}{$\begin{array}{l}\mathrm{P} 1- \\
\text { Entrée dans le } \\
\text { processus }\end{array}$} & \multicolumn{2}{|c|}{$\begin{array}{c}\mathrm{P} 2- \\
\text { Ancrage dans le } \\
\text { processus }\end{array}$} & \multicolumn{2}{|c|}{ Évolution } \\
\hline Axes & Ampleur & Fidélité & Ampleur & Fidélité & Ampleur & Fidélité \\
\hline Stratégie et gouvernance & 3 & 3 & 3 & 3 & -- & -- \\
\hline $\begin{array}{l}\text { Politique sociale et ancrage } \\
\text { territorial }\end{array}$ & 1,5 & 3 & 3 & 2 & $+1,5$ & -1 \\
\hline Gestion environnementale & 2 & 2 & 2 & 4 & - & +2 \\
\hline $\begin{array}{l}\text { Total ampleur / } \\
\text { total fidélité }\end{array}$ & 6,5 & 8 & 8 & 9 & $+1,5$ & +1 \\
\hline $\begin{array}{l}\text { 8. Université Sud } \\
\text { Toulon - Var }\end{array}$ & \multicolumn{2}{|c|}{$\begin{array}{c}\mathrm{P} 1- \\
\text { Entrée dans le } \\
\text { processus }\end{array}$} & \multicolumn{2}{|c|}{$\begin{array}{c}\mathrm{P} 2- \\
\begin{array}{c}\text { Ancrage dans le } \\
\text { processus }\end{array}\end{array}$} & \multicolumn{2}{|c|}{ Évolution } \\
\hline Axes & Ampleur & Fidélité & Ampleur & Fidélité & Ampleur & Fidélité \\
\hline Stratégie et gouvernance & 2 & 2 & 1 & 2 & -1 & -- \\
\hline $\begin{array}{l}\text { Politique sociale et ancrage } \\
\text { territorial }\end{array}$ & 1,5 & 3 & 2 & 3 & $+0,5$ & -- \\
\hline Gestion environnementale & 1 & 1 & 1 & 2 & - & +1 \\
\hline $\begin{array}{l}\text { Total ampleur / } \\
\text { total fidélité }\end{array}$ & 4,5 & 6 & 4 & 7 & $-0,5$ & +1 \\
\hline
\end{tabular}




\begin{tabular}{|l|c|c|c|c|c|c|}
\hline $\begin{array}{c}\text { 9. Université } \\
\text { professionnelle } \\
\text { Internationale Lyon } \\
\text { René Cassin }\end{array}$ & \multicolumn{2}{|c|}{$\begin{array}{c}\text { P1- } \\
\text { Entrée dans le } \\
\text { processus }\end{array}$} & $\begin{array}{c}\text { Axes } \\
\text { Ancrage dans le } \\
\text { processus }\end{array}$ & \multicolumn{2}{c|}{ Évolution } \\
\hline Ampleur & Fidélité & Ampleur & Fidélité & Ampleur & Fidélité \\
\hline Stratégie et gouvernance & 3 & 3 & 2 & 4 & -1 & +1 \\
\hline $\begin{array}{l}\text { Politique sociale et ancrage } \\
\text { territorial }\end{array}$ & 2 & 3 & 2 & 3 & -- & - \\
\hline Gestion environnementale & 3 & 4 & 2 & 3 & -1 & -1 \\
\hline $\begin{array}{l}\text { Total ampleur / total } \\
\text { fidélité }\end{array}$ & 8 & 10 & 6 & 10 & -2 & -- \\
\hline
\end{tabular}

Au cours de cette section, nous avons présenté le rôle du champ institutionnel dans le processus d'adoption des rapports développement durable par les universités, nous avons décrit les thèmes du référentiel Plan Vert et la collecte de nos données. Les tableaux de synthèse ci-avant sont le résultat de l'observation et du recueil des données relatifs au reporting développement durable de neuf universités. Comme nous l'avons expliqué précédemment, nous les avons réparties en deux groupes pionniers : les innovateurs et les adoptants précoces. Chacun des groupes, observés selon un double prisme - temporel et ampleur/fidélité des pratiques de reporting - révèle des enseignements intéressants. Nous les présentons et les discutons dans la section suivante.

\section{Discussion}

L'initiative Campus Responsable, suivie, affinée et systématisée par le référentiel «Plan Vert » constitue pour les établissements d'enseignement supérieur un outil relativement souple qui présente les caractéristiques énoncées par Ansari \& al. (2010) :

- une interprétativité élevée car bien qu'il résulte d'un dispositif législatif, il offre des possibilités d'adaptation multiples aux établissements ;

- une divisibilité importante puisqu'il ne doit pas obligatoirement être adopté sur toutes ses dimensions et que chacun de ses items peut faire l'objet d'une adoption plus ou moins importante ;

- une complexité forte générée par la variété et l'ampleur des sujets inclus dans le Plan Vert, en même temps que par la diversité des parties prenantes impliquées.

Ces caractéristiques vont conduire les établissements à décliner l'outil lors de sa mise en œuvre de façon plus ou moins fidèle et ample, sans que l'une ou l'autre de ces dimensions apparaisse prépondérante a priori. Il nous semble donc particulièrement intéressant d'observer les modalités de cette déclinaison dans les établissements pionniers (constitués des innovateurs et des adoptants précoces dont les pratiques de reporting développement durable perdurent) que nous avons identifiés. 
Figure 2 : Évolution de la fidélité et de l'ampleur des pratiques organisationnelles des innovateurs entre la période d'entrée dans le processus de reporting et celle d'ancrage dans ce processus

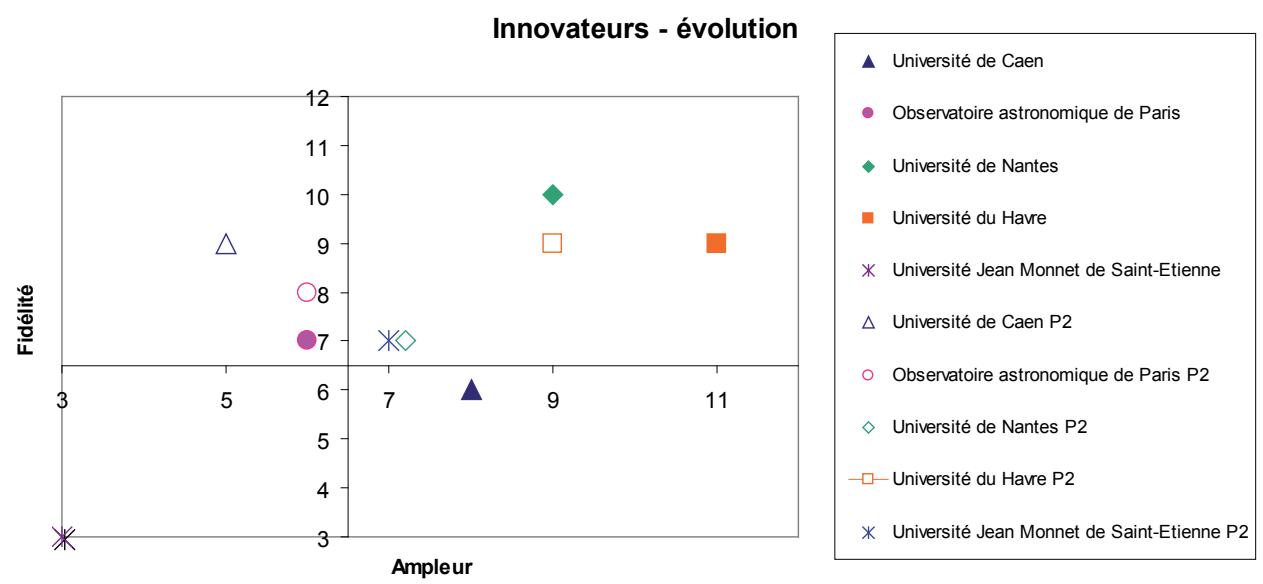

Ainsi, en observant la position initiale des universités appartenant au groupe des innovateurs (figure 2, signes pleins), on voit que 3 universités sur 5 se situent dans les cadrans « adaptation complète et fidèle » (2) et « adaptation sur mesure » (1). De même, 3 universités sur 5 montrent un bon niveau de fidélité. Cela paraît cohérent avec la démarche de ces acteurs : innovants, ils s'appliquent très tôt à renseigner à la fois plus complètement et de façon plus intense les grilles de reporting. Plus surprenant, une université, bien qu'innovatrice, affiche une adaptation très distante de cette pratique tant sur l'axe de la fidélité que sur celui de l'ampleur.

Lorsque ces universités innovantes confirment leur engagement (figure 2, signes vides), on constate qu'elles montrent toutes (sauf une), une fidélité en progression. Cela se manifeste par un net investissement des cadrans supérieurs de la figure, sur les zones « adaptation complète et fidèle » (3) et « adaptation à faible dose » (2). En termes d'ampleur, les membres du groupe sont assez proches les uns des autres puisqu'ils obtiennent des notes comprises entre 5 et 9 . Plus aucune université ne se trouve dans le cadran en bas à gauche qualifié d' « adaptation distante ».

Les bons « résultats » des innovateurs (universités situées dans les cadrans supérieurs) de la période 2 s'expliquent sans doute par la forte congruence technique entre les pratiques de communication en développement durable (reporting) et les organisations universitaires adoptantes, ces dernières ayant l'habitude de rendre des comptes à de nombreuses parties prenantes, tant pour leur fonctionnement (par exemple au travers de leurs contrats quadriennaux / quinquennaux) que pour la reconduction de leurs diplômes ou l'évaluation des enseignants-chercheurs.

Du point de vue de l'évolution de l'engagement des innovateurs entre la période 1 et la période 2, nous pouvons dire que, globalement, les innovateurs gagnent en fidélité (avec cependant des évolutions contrastées) et perdent en ampleur. Autrement dit, cette population, qui a devancé l'appel de la loi et de la norme, évolue en produisant un reporting plus complet sur les dimensions constitutives des (trois) thématiques associées au développement durable. En revanche, le degré d'intensité des pratiques (ampleur) décroît pour les universités de ce groupe, hormis l'université de Saint-Étienne qui était très en retard et qui 
comble ses lacunes en rejoignant les autres innovateurs en période 2. Ces conclusions ne sont pas surprenantes. En effet, en entrant précisément dans les reportings développement durable de nos innovateurs, on perçoit une grande divisibilité (augmentation du nombre d'items qui ne peuvent être tous renseignés). Cela se traduit par un investissement qui peut sembler moins intense (ampleur moindre). En revanche, en s'ancrant dans la pratique de reportings développement durable, les innovateurs font face à une complexité accrue. Ils sont alors contraints à une adaptation très marquée en termes de fidélité mais doivent amoindrir leurs exigences en termes d'ampleur. Cela se passe comme si le groupe des innovateurs ne pouvait pas, dans un temps court, être à la fois pionnier et s'investir simultanément avec la même force sur les deux axes, ce qui confirme l'hypothèse d'Ansari \& al. selon laquelle une grande divisibilité permettrait une adaptation réduite dans son ampleur mais n'affecterait pas la fidélité d'une pratique.

La congruence politique (compatibilité des caractéristiques normatives de la nouvelle pratique avec les intérêts et priorités des organisations adoptantes) faible entre les pratiques de communication en développement durable et les organisations universitaires qui les mettent en place nous semble un facteur explicatif : le premier référentiel français de développement durable applicable aux établissements d'enseignement supérieur et de recherche n'a en effet été finalisé qu'en octobre 2009 et son lancement officiel, début 2010 , est vraisemblablement passé au second plan alors que les établissements français devaient, dans le même temps, intégrer la loi du 10 août 2007 relative aux libertés et responsabilités des universités ${ }^{5}$ et passer progressivement aux «Responsabilités et Compétences Élargies $»^{6}$. Le déplacement de l'énergie (plus de fidélité au prix de moins d'ampleur) constaté est donc probablement dû à des choix d'agendas politiques. De plus, l'accroissement de la fidélité allié à une dimension relative de l'ampleur peut être lu comme une volonté de communiquer sur des sujets plus variés, superficiellement plutôt qu'en profondeur.

Observons maintenant le comportement des adoptants précoces (figure 3).

De la même manière, en observant la position initiale des universités appartenant au groupe des adoptants précoces (signes pleins), on peut avancer que, d'une part, de façon générale, il n'y a pas d'individu très atypique. D'autre part, les individus se situent plutôt dans les cadrans supérieurs (ou très proche), ce qui signifie que la fidélité est satisfaisante, voire bonne. Enfin, l'ampleur constatée est assez variable (pas de tendance nette), mais peu dispersée (entre 4,5 et 8/12)

Lorsque ces mêmes universités confirment leur engagement (période d'ancrage, figure 3 , signes vides), on constate qu'elles occupent toutes clairement les cadrans supérieurs, avec de bonnes fidélités peu dispersées (notes entre 7 et 10/12). L'ampleur est, elle, assez limitée, et comme en période d'entrée, peu dispersée (entre 4 et 8/12).

Les « résultats » satisfaisants des adoptants précoces (universités situées dans les cadrans supérieurs) que ce soit en période 1 ou 2 trouvent leur justification dans une bonne congruence technique entre les pratiques de communication en développement durable (reporting) et les organisations universitaires. En effet, comme le groupe des universités « innovatrices », elles ont l'habitude de rendre des comptes et le référentiel Plan Vert ne constitue pas, en ce sens, un outil original. Il les surprend d'autant moins qu'elles l'ont vu pratiquer par les universités « innovatrices ». Nous sommes ici face à l'expression de la transmission normative combinée au mimétisme.

\footnotetext{
${ }^{5}$ J.O. $n^{\circ} 185$ du 11 août 2007, page 13468 texte $n^{\circ} 2$.

6 Nouveau cadre de gestion qui modifie les règles et modes de gestion financière et de gestion des ressources humaines des universités françaises ainsi que leurs fonctions de direction et d'aide au pilotage.
} 
Figure 3 : Évolution de la fidélité et de l'ampleur des pratiques organisationnelles des adoptants précoces entre la période d'entrée dans le processus de reporting et celle d'ancrage dans ce processus

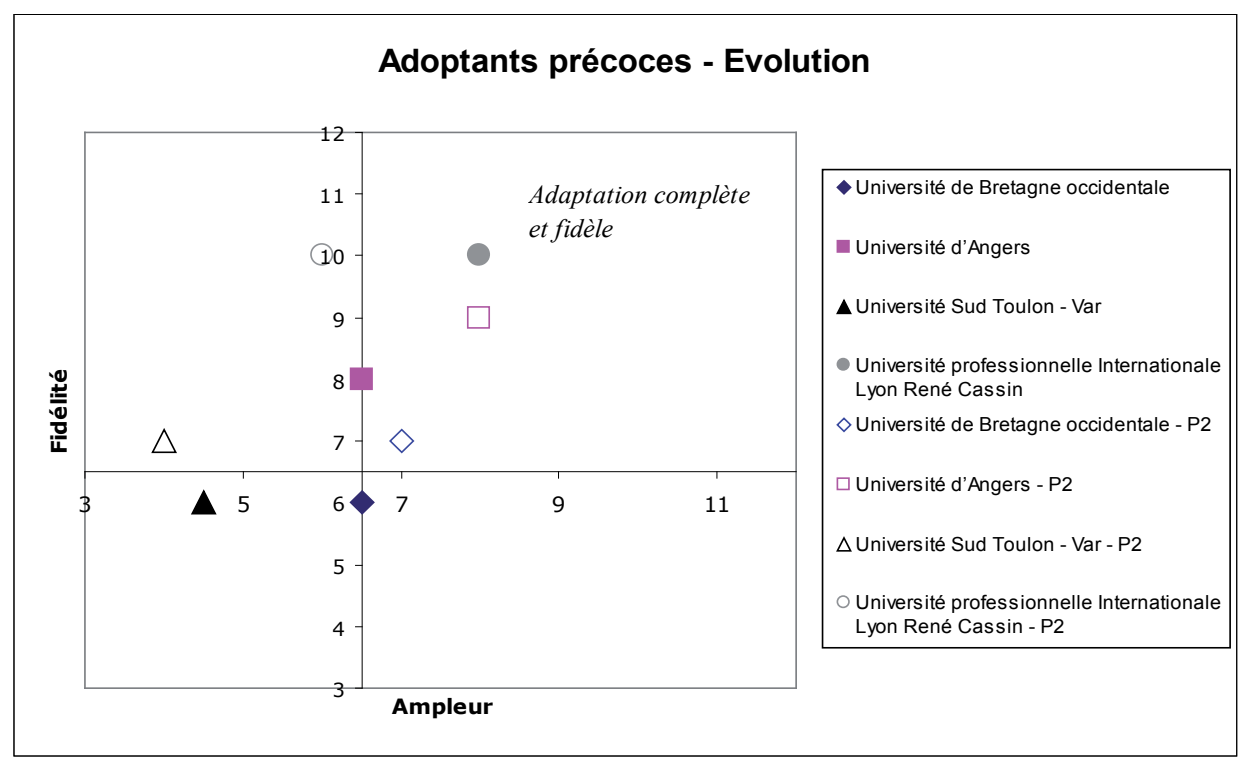

Du point de vue de l'évolution de l'engagement du groupe des «adoptants précoces » entre les deux périodes, nous pouvons dire que globalement, les universités progressent sur l'axe de fidélité tandis qu'elles évoluent sans tendance nette sur l'axe ampleur. Il semble que les rapports développement durable mettent en évidence des pratiques sur un plus grand nombre de dimensions (fidélité) sans montrer clairement une amélioration de leur intensité (deux universités progressent en termes d'ampleur du reporting, tandis que deux décroissent).

Ces résultats pourraient être interprétés ainsi : la population des « adoptants précoces », qui bénéficie d'un effet d'expérience grâce à l'observation du groupe des « innovateurs » qui l'a précédée produit un reporting plus complet sur les dimensions constitutives des (trois) thématiques associées au développement durable. En revanche, le degré d'intensité des pratiques (ampleur) est sensiblement similaire entre les deux périodes, et globalement moins bon que celui des « innovateurs ». Les adoptants précoces rompus à l'exercice de reporting en tout genre et pas réellement novices en termes de communication développement durable parviennent à instrumentaliser l'outil : ils renseignent des items sur chacun des thèmes mais s'investissent modérément en termes d'ampleur. Comme pour le groupe des « innovateurs », les « adoptants précoces » ont choisi de privilégier l'axe de la fidélité, plus rapidement valorisable et valorisante. On peut se demander si l'amélioration de la « fidélité » ne correspond pas, au moins en partie, à une conformation croissante du rapport aux attentes présumées des parties prenantes (phénomène de «routinisation » du reporting).

En comparant les figures 2 et 3 , on constate que, globalement, nos groupes ont des comportements différents. En effet, les « innovateurs » affichent de meilleures performances tant en termes de fidélité que d'ampleur (sauf un individu en période 1), avec des notes maximales de fidélité s'élevant à 10/12 et d'ampleur à 11/12. Les « adoptants précoces » ont également de très bonnes performances de fidélité mais modèrent leur engagement en 
termes d'ampleur. Sans doute que la congruence politique faible (qui ne s'améliore pas pour les « adoptants précoces ») associée à une congruence culturelle également limitée entre les pratiques de communication en développement durable et les universités constitue une double explication. Nous avons déjà vu ci-dessus que nous pouvions considérer la congruence politique comme peu assurée. Quant à la congruence culturelle (compatibilité de la nouvelle pratique avec les valeurs, croyances et pratiques mobilisées par les organisations adoptantes), celle-ci ne nous semble guère plus importante car, si la plupart des questions relatives au développement durable ont déjà fait l'objet de nombreuses recherches et d'enseignement, les implications de ces questions sur les établissements eux-mêmes et leurs campus ne recevaient jusqu'à récemment qu'une faible attention (College Sustainability Report Card 2007) même si celle-ci semble cependant croître peu à peu. Les organisations universitaires subissent par conséquent de plus en plus de contraintes qui placent le reporting développement durable dans la « file d'attente » des devoirs et obligations. Par ailleurs, une divisibilité croissante de l'outil ainsi qu'une augmentation de sa complexité justifient également les hauts niveaux de fidélité couplés à des niveaux plus mesurés d'ampleur, ce qui est cohérent avec les hypothèses d'Ansari et al.

Dans ce contexte contraignant, les universités confirment leur communication en développement durable sur des thèmes visibles et aisés à transcrire, mais n’ont pas les moyens, dans l'immédiat, d'approfondir ou améliorer la pratique. 
Finalement, en comparant les variations relatives du groupe des innovateurs (figure 4) et du groupe des adoptants précoces (figure 5) entre l'entrée et l'ancrage dans la pratique de reporting, on visualise aisément que les variations sont assez importantes, tant en termes de fidélité (variations positives) que d'ampleur (variations globalement négatives), pour les universités « innovatrices » tandis que les variations sont faibles, tant en termes de fidélité (variations légèrement positives) que d'ampleur (variations légèrement positives), pour les universités « adoptants précoces ». Cette double analyse confirme nos observations précédentes.

Figure 4 : Variations relatives de la fidélité et de l'ampleur des pratiques organisationnelles des innovateurs entre la période d'entrée dans le processus de reporting et celle d'ancrage dans ce processus

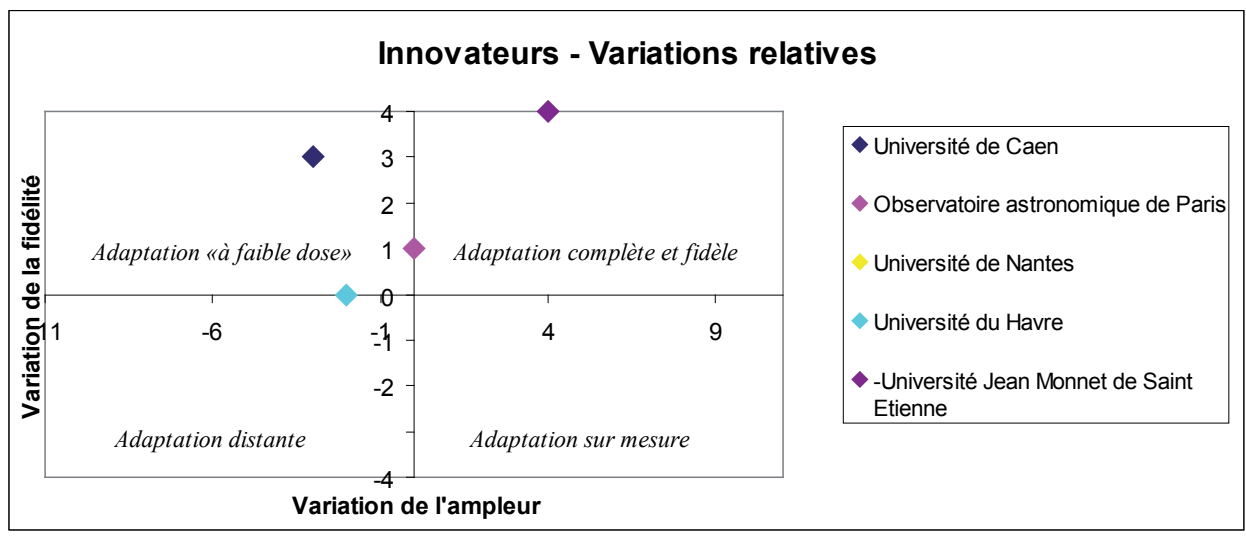

Figure 5 : Variations relatives de la fidélité et de l'ampleur des pratiques organisationnelles des adoptants précoces entre la période d'entrée dans le processus de reporting et celle d'ancrage dans ce processus

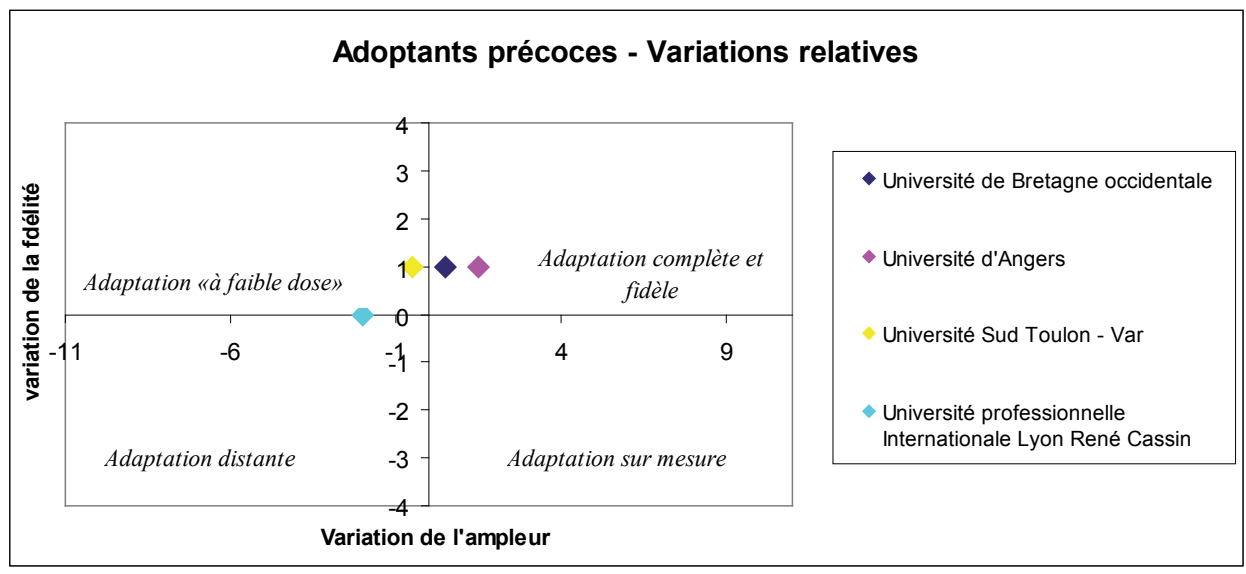




\section{Conclusion}

L'objectif de notre contribution était d'observer l'adoption et la diffusion d'une pratique organisationnelle relativement récente par (et dans) les établissements français d'enseignement supérieur : le reporting développement durable. Pour répondre à notre question - Comment les établissements français d'enseignement supérieur manifestent-ils l'adoption et la diffusion d'une nouvelle pratique organisationnelle ? - nous avons évalué l'évolution de la communication des universités selon deux dimensions : l'ampleur et la fidélité du reporting.

Nous avons examiné notamment quels critères économiques, sociaux et environnementaux étaient renseignés par les universités pionnières pour manifester l'adoption et la diffusion du développement durable dans et à l'extérieur de leur organisation. L'inscription dans le champ institutionnel, qui augmente la coercition, mais également la transmission normative et le mimétisme, peut expliquer la confirmation et l'évolution du reporting. De même, l'évolution des pratiques de communication du développement durable par les universités, qu'elles soient « innovateurs » ou « adoptants précoces » semble contingente de la bonne congruence technique, mais également des faibles congruences politique et culturelle.

$\mathrm{Au}$ total, il semble que nos acteurs universitaires satisfont aux injonctions du champ institutionnel et des parties prenantes en répondant de façon satisfaisante aux dimensions de fidélité et d'ampleur du référentiel Plan Vert. Cela dit, si les universités pionnières étudiées satisfont formellement aux différents critères et obtiennent alors de bonnes évaluations, une lecture attentive de leurs reportings laisse penser que les notes obtenues ne préjugent en rien de l'engagement réel en développement durable ${ }^{7}$. Cette dernière remarque est inspirée des travaux de Westphal et Zajac $(1994,1998)$. En effet, si le reporting développement durable, imposé par le champ institutionnel agit comme une démarche de légitimation vis-à-vis des parties prenantes, il est fréquent d'opérer une distinction entre contrôle des prestations et évaluation des résultats (Varone, 2008), adoption d'une pratique et mise en œuvre. Cette déconnexion soulignée par nombre d'auteurs semble pouvoir être étendue au reporting développement durable. Un lien pourrait ici être réalisé avec les limites inhérentes au principe d'accountability (Messner, 2009) et notamment au fait qu'il semble délicat de réduire la notion d'accountability à la justification de ses actions pour son intérêt propre.

\section{Intérêts et limites de la recherche}

Le premier intérêt de notre travail est de s'être penché sur une pratique organisationnelle encore relativement récente dans les universités françaises en étudiant la communication en développement durable des établissements pionniers.

Le deuxième élément saillant est l'approche comparative que nous avons privilégiée. En effet, nous avons choisi de comparer les « innovateurs » et les « adoptants précoces » entre eux (intergroupe) mais aussi au sein de leur propre famille (intragroupe), ainsi que sur deux périodes (période d'entrée dans le processus (adoption) et période d'ancrage de la pratique de reporting (diffusion)).

\footnotetext{
${ }^{7}$ Une perspective d'évolution de cette recherche pourrait être d'évaluer les pratiques de développement durable par rapport aux pratiques de reporting développement durable. Il serait alors intéressant d'examiner dans quelle mesure le constat de « normalisation » progressive des rapports au fil du temps, qui accroît optiquement la fidélité, s'accompagne - ou non - d’un écart croissant avec la réalité.
} 
Le troisième point notable est la volonté d'inscrire cette recherche dans une logique cumulative : pour observer l'adoption et la diffusion des pratiques de reporting, nous avons délibérément opté pour les deux dimensions - ampleur et fidélité - éprouvées par Ansari $\&$ al. (2010) lors de leur analyse de l'inscription des pratiques organisationnelles. Les résultats que nous obtenons, bien que relativement modestes, sont cohérents et porteurs de perspectives de recherches futures.

Notre recherche revêt des limites inhérentes à notre terrain. En effet, nous n'avons pu observer qu'un petit nombre d'universités car notre volonté était d'étudier les établissements qui réalisaient un reporting développement durable récurrent. Aujourd'hui, nous l'avons vu, seules cinq universités appartiennent au groupe des « innovateurs » (elles ont fait acte de communication dès la première période et poursuivent leur reporting aujourd'hui) et quatre sont des «adoptants précoces » (elles ont communiqué leur engagement en développement durable un peu plus tard et continuent à répondre au référentiel actuellement). En outre, nos choix empiriques ne nous permettent pas de préciser ici les conditions d'adoption locales du reporting (quelles parties prenantes ? quelle majorité ? quelles motivations ?....). Ces éléments pourraient être étudiés lors d'un travail complémentaire.

La récence de l'adoption du reporting développement durable explique également le relativement faible recul dont nous disposons pour élaborer des conclusions qui nous permettraient de nous projeter dans l'avenir et émettre d'ores et déjà des recommandations.

\section{Perspectives de la recherche}

Ce travail de recherche est la première pierre d'un édifice en construction. Nous prévoyons de poursuivre notre analyse comparative (intergroupe, intragroupe et longitudinale) en intégrant un plus grand nombre d'universités, au fil de l'adoption et de la diffusion de leur pratique de reporting. Cette évolution de notre travail peut nous faire espérer des conclusions intéressantes sur les lois de la diffusion des pratiques nouvelles dans un corps social donné. Nous espérons ainsi non seulement augmenter notre taille d'échantillon, mais également améliorer notre approche longitudinale (espace temporel allongé) et finalement constituer des groupes d'entrants, selon la nomenclature de Rogers (1995). Dans cet esprit, nous observerons avec le plus grand intérêt l'arrivée du groupe de la « majorité » dans le panorama. Comment se groupe se comportera-t-il sur les dimensions de l'ampleur et de la fidélité ? Quelles seront, à cette date, les congruences technique, politique et sociale entre la pratique de reporting et les universités ?

Par ailleurs, l'arrivée des universités de la « majorité » nous renvoie au « chasm» (l'abîme) de Moore (1999), situé entre les « adoptants précoces » et la « majorité avancée ». En effet, le «chasm » représente le passage d'un marché de niche à un marché de masse. Or, Rogers nous a appris que les attentes des consommateurs sont très différentes entre ces deux types d'acteurs. Les « innovateurs » et les « adoptants précoces » sont des acteurs enthousiastes, relativement faciles à convaincre. À l'inverse, les acteurs de la majorité sont des pragmatiques. À l'instar de la théorie de la diffusion de l'innovation, on passerait d'universités sensibles et enthousiastes à la nouvelle pratique organisationnelle de reporting développement durable, à des établissements plus prudents, voulant des preuves tangibles de l'utilité et des performances de l'outil, et attendant qu'un référentiel universel et définitif soit choisi. Ainsi, dès l'entrée de la « majorité » et par son impulsion, les degrés d'interprétativité, de divisibilité et de complexité de l'outil devraient s'amoindrir, ce qui 
aboutirait à une homogénéisation des pratiques des acteurs universitaires. Face à une professionnalisation croissante du champ, les pressions normatives seraient ici prépondérantes.

Les voies de recherche que nous venons de présenter pourraient également être étendues à l'échantillon des écoles d'ingénieur et de commerce, institutions d'enseignement supérieur spécifiques à la France mais très présentes (en nombre et en qualité) dans le paysage de la formation supérieure.

\section{Bibliographie}

ANSARI S.M., FISS P.C., ZAJACE.J., (2010). Made to fit: how practices vary as they diffuse, Academy of Management Review 35(1), 67-92.

BENDERS J., VAN VEEN K., (2001). What's in a fashion? Interpretative viability and management fashions, Organization 8, 33-53.

BLEIKLIE I., POWELL W.W., (2005). Universities and the production of knowledge - Introduction, Higher Education 49, 1-8.

CAPRON M., (2004). L'inscription de l'enseignement et de la recherche sur la RSE dans le contexte politique français et européen, La Revue des Sciences de Gestion 205, 117-124.

CAPRON M., QUAIREL-LANOIZELÉE F., (2007). La responsabilité sociale d'entreprise, La Découverte, Paris.

CASTELNAU P., NOËL C., (2004). Engagement pour un développement durable et performance des entreprises : le cas français, Journées du CERMAT sur la performance, IAE de Tours.

CLARKSON M.B., (1995). A Stakeholder Framework for Analyzing and Evaluating Corporate Social Performance, Academy of Management Review 20 (1), 92-117.

COLLIER P.M., (2001). The power of accounting: a field study of local financial management in a police force, Management Accounting Research 12, 465-486.

COOPER S.M., OWEN D.L., (2007). Corporate social reporting and stakeholder accountability: The missing link, Accounting, Organizations and Society 32 (7-8), 649-667.

DÉJEAN F., OXIBAR B., (2005). Pratiques de diffusion d'information sociétale : Le cas Péchiney, Journée Franco-Espagnole de comptabilité - Les nouvelles comptabilités : recherche, enseignement, pratique, Université Paris Dauphine.

DEMEESTERE R.,ORANGE G., (2008). Gestion publique : qu'est-ce qui a changé depuis 25 ans ?, Politiques et Management Public 26 (3), 127-147.

DIMAGGIO P.J., POWELL W.W., (1983). The Iron Cage Revisited: Institutional Isomorphism and Collective Rationality in Organizational Fields, American Sociological Review 48, 147-160.

DONALDSON T., PRESTON L.E., (1995). The Stakeholder Theory of the Corporation: Concepts, Evidence and Implications, Academy of Management Review 20 (1), 65-91.

DOWLING J., PFEFFER J., (1975). Organizational legitimacy: Social values and organizational behaviour, Pacific Sociological Review 18, 122-136.

FREEMAN R.E., (1984). Strategic Management: A Stakeholder Approach, Pitman, Boston.

HASSELBLADH H., KALLINIKOS J., (2000). The project of rationalization: a critique and reappraisal of neoinstitutionalism in organization studies, Organization Studies 21 (4), 697-720.

LACASSE F., VERRIER P.-E., (2005). 30 ans de réforme de l'État, expériences françaises et étrangères : stratégie et bilans, Dunod, Paris.

LAPSLEY I., (2008). The NMP agenda: back to the future, Financial Accountability \& Management 24, 77-96.

LAPSLEY I., (2009). New Public Management: The Cruellest Invention of the Human Spirit?, Abacus 45, 1-21. 
LAUFER R., (1996). Quand diriger, c'est légitimer, Revue Française de Gestion 111, 12-37.

LOZANO R., (2010). Diffusion of sustainable development in universities' curricula: an empirical example from Cardiff University, Journal of Cleaner Production 18, (7), 637-644.

MERLIN-BROGNIART C., DEPRET M.-H., (2009). Quels acteurs pour quelle responsabilité des entreprises ? D'une responsabilité sociale des entreprises à la responsabilité globale des acteurs, 30 avril, conférence RIODD.

MESSNER M., (2009). The limits of accountability, Accounting, Organizations and Society 34(8), 918-938.

MEYER J.W., ROWAN B., (1977). Institutionalized Organizations: Formal Structure as Myth and Ceremony, American Journal of Sociology, 340-363.

MILES M.B., HUBERMAN A. M., (1994). Qualitative Data Analysis (2 $2^{\text {nd }}$ ed), Sage, Thousand Oak.

MITCHELL R.K., AGLE B.R., WOOD D.J., (1997). Toward a Theory of Stakeholder Identification and Salience : Defining the Principle of Who and What Really Counts, Academy of Management Review 22 (4), 853-886.

MOORE G., (1999). Tinged Shareholder Theory: Or what's so special about stakeholders?, Business Ethics: A European Review 8 (2), 117-127.

POLLIT C., (2003). The Essential Public Manager, Open University Press, Berkshire.

PFEFFER J., SALANCIK G.R., (1978). The external control of organizations : a resource dependence perspective, Harper \& Row, New York.

PHILLIPS N., LAWRENCE T., HARDY C., (2004). Discourse and institutions, Academy of Management Review 29 (4), 635-652.

RICHARDSON A.J., (1987). Accounting as a legitimating institution, Accounting Organization and Society 12 (4), 341-355.

ROGERS E.M., (1962). Diffusion of innovations (1 ${ }^{\text {rst }}$ ed.), Free Press, NewYork.

ROGERS E.M., (1983). Diffusion of innovations ( $3^{\text {rd }}$ ed.), Free Press, NewYork.

ROGERS E.M., (1995). Diffusion of innovations (4 ${ }^{\text {th }}$ ed.), Free Press, NewYork.

SCOTT W.R., (1995). Institutions and organizations, Sage, Thousand Oak.

SCOTT W.R., (2001). Institutions and organizations ( $2^{\text {nd }}$ ed.), Sage, Thousand Oak.

SHOCKER A.D., SETHI S.P. (1974) An approach to incorporating social preferences in developing corporate action strategies, In Sethi S.P (dir.) The unstable groung : Corporate social policy in a dynamic society, Melville, CA.

SUCHMAN M.C., (1995). Managing Legitimacy: Strategic and Institutional Approaches”, Academy of Management Journal 20 (3), 571-610.

VARONE F., (2008). De la performance publique: concilier évaluation des politiques et budget par programme ?, Politiques et Management Public 26 (3), 77-89.

WESTPHAL J.D., ZAJAC E.J., (1994). Substance and symbolic in CEOs' Long-term Incentive Plans, Administrative Science Quarterly, 367-390.

WESTPHAL J.D., ZAJAC E.J., (1998). The symbolic management of stockholders : corporate governance reforms and shareholder reactions, Administrative Science Quarterly, 127-153.

ZALTMAN G., DUNCAN R., HOLBECK J., (1973). Innovation and organizations, John Wiley, Chischester. 


\section{Annexe \\ Grille de synthèse du reporting de l'université de Nantes (groupe des « innovateur »)}

\begin{tabular}{|c|c|c|}
\hline \multicolumn{3}{|c|}{ Thématique «Stratégie et gouvernance » } \\
\hline Dimensions & $\begin{array}{c}\text { P1 } \\
\text { Entrée dans le processus }\end{array}$ & $\begin{array}{c}\mathrm{P} 2 \\
\text { Ancrage dans le processus }\end{array}$ \\
\hline $\begin{array}{l}\text { Engagement } \\
\text { de la présidence }\end{array}$ & $\begin{array}{l}\text { - Formalisation de la lettre de mission du } \\
\text { «Conseiller Université } 21 » \text { qui lui donne } \\
\text { une compétence transversale pour bâtir, } \\
\text { proposer au Conseil d'Administration et } \\
\text { contrôler la stratégie globale en matière de } \\
\text { développement durable. Le cadre en a été } \\
\text { accepté lors de l'élection du Bureau qui } \\
\text { assiste le président de l'Université dans } \\
\text { le pilotage de l'établissement durant son } \\
\text { mandat (cinq ans). }\end{array}$ & $\begin{array}{l}\text { - Mise en place d'un Agenda } 21 . \\
\text { - Une équipe mission Université } \\
21 \text { avec un coordinateur et un } \\
\text { conseiller au sein du CA de } \\
\text { l'université (5h/semaine). }\end{array}$ \\
\hline $\begin{array}{l}\text { Prospective, } \\
\text { planification } \\
\text { et réalisation }\end{array}$ & $\begin{array}{l}\text { - Établissement d'un bilan annuel et d'un } \\
\text { compte-rendu des activités transversales de } \\
\text { développement durable devant le Conseil } \\
\text { d'Administration tous les deux mois. }\end{array}$ & \\
\hline $\begin{array}{l}\text { Gouvernance } \\
\text { participative }\end{array}$ & $\begin{array}{l}\text { - Mise en place d'un «Conseiller Université } \\
21 » \text { et d'un réseau développement durable } \\
\text { composé d'un représentant par Unité de } \\
\text { Formation et de Recherche (UFR) servant de } \\
\text { relais au «Conseiller Université } 21 » . \\
\text { - Organisation de nombreux événements de } \\
\text { sensibilisation : projection débat du film } \\
\text { d'Al Gore avec débat sur les implications } \\
\text { pour l'Université de ces enjeux sur tous les } \\
\text { sites (six, dont trois villes différentes), une } \\
\text { conférence par mois au sein de toutes les } \\
\text { UFR sur les implications des changements } \\
\text { nécessaires sur la vie quotidienne des } \\
\text { universités. } \\
\text { - Consultation des parties prenantes par } \\
\text { l'intermédiaire d'un correspondant } \\
\text { enseignant par UFR et d'un correspondant } \\
\text { IATOS (ingénieurs et agents techniques, } \\
\text { ouvriers et de services) par bâtiment. }\end{array}$ & $\begin{array}{l}\text { - Comité de pilotage } \\
\text { développement durable avec } \\
\text { des personnels enseignants, } \\
\text { administratifs et techniques, } \\
\text { et des étudiants des différents } \\
\text { campus ( } 4 \text { réunions par an). }\end{array}$ \\
\hline Efficacité budgétaire & & \\
\hline \multicolumn{3}{|c|}{ Thématique « Politique sociale et ancrage territorial» } \\
\hline Dimensions & $\begin{array}{c}\text { P1 } \\
\text { Entrée dans le processus }\end{array}$ & $\begin{array}{c}\text { P2 } \\
\text { Ancrage dans le processus }\end{array}$ \\
\hline Équité sociale & $\begin{array}{l}\text { - Accompagnement des handicapés physiques } \\
\text { par des tuteurs. } \\
\text { - Présence d'équipements facilitant } \\
\text { l'accessibilité (rampes, ascenseurs). } \\
\text { - Appui de l'association Handisup qui développe } \\
\text { des moyens propres à compenser les situations } \\
\text { de handicap des étudiants inscrits. }\end{array}$ & $\begin{array}{l}\text { - Diversité étudiante : plus de } \\
30 \% \text { de boursiers du CROUS. }\end{array}$ \\
\hline
\end{tabular}




\begin{tabular}{|c|c|c|}
\hline $\begin{array}{l}\text { Développement } \\
\text { individuel }\end{array}$ & $\begin{array}{l}\text { - Entretiens individualisés sur l'évolution } \\
\text { et l'évaluation des fonctions du personnel } \\
\text { avant la notation. } \\
\text { - Participation à des échanges de type } \\
\text { coopération ou solidarité active : } \\
\text { par exemple, une aide est apportée } \\
\text { au Groupement étudiant national } \\
\text { d'enseignement aux personnes incarcérées } \\
\text { (GENEPI) de la part de la faculté de droit. }\end{array}$ & $\begin{array}{l}\text { - Environ } 10 \% \text { d'étudiants } \\
\text { étrangers chaque année. } \\
\text { Organisation d'un guichet } \\
\text { unique par l'université pour } \\
\text { leur inscription et leurs } \\
\text { démarches administratives. } \\
\text { - Soutien financier aux } \\
\text { associations étudiantes : } \\
\text { «les Éveilles » organisent } \\
\text { des bourses aux livres et des } \\
\text { petits-déjeuners bio / équitable, } \\
\text { « Bullivers » a créé un potager } \\
\text { partagé sur le campus sciences } \\
\text { et organise des projections / } \\
\text { débats sur la thématique de } \\
\text { l'alimentation durable, et } \\
\text { «Et si on s'activait » a organisé } \\
\text { un festival ouvert au public } \\
\text { sur l'écologie au quotidien. } \\
\text { - Organisation par la mission } \\
\text { Université } 21 \text { de projections, } \\
\text { débats, conférences sur le } \\
\text { développement durable } \\
\text { ouverts au public. } \\
\text { - Formation des acheteurs de } \\
\text { l'université au développement } \\
\text { durable. }\end{array}$ \\
\hline Ancrage territorial & $\begin{array}{l}\text { - Plateforme du développement durable, } \\
\text { mise en réseaux, projet de fondation } \\
\text { en partenariat avec la Région, Nantes } \\
\text { Métropole et le Centre National de } \\
\text { Recherche Scientifique (CNRS). }\end{array}$ & \\
\hline Efficacité budgétaire & & \\
\hline \multicolumn{3}{|c|}{ Thématique « Gestion environnementale » } \\
\hline Dimensions & $\begin{array}{l}\text { P1 - } \\
\text { Entrée dans le processus }\end{array}$ & $\begin{array}{l}\mathrm{P} 2- \\
\text { Ancrage dans le processus }\end{array}$ \\
\hline $\begin{array}{l}\text { Stratégie de } \\
\text { l'établissement }\end{array}$ & - Projet de réalisation d'un Bilan Carbone ${ }^{\circledR}$. & $\begin{array}{l}\text { - Signature de la Déclaration } \\
\text { des campus responsables } \\
\text { pour le climat. } \\
\text { - Réalisation des Bilans } \\
\text { Carbones® de deux sites } \\
\text { en } 2009 \text { / } 2010 \text {. }\end{array}$ \\
\hline $\begin{array}{l}\text { Gestion des } \\
\text { ressources et intrants }\end{array}$ & $\begin{array}{l}\text { - Intégration du développement durable } \\
\text { dans tous les postes d'achats. }\end{array}$ & $\begin{array}{l}\text { - Mise en place d'une politique } \\
\text { d'achats responsables. }\end{array}$ \\
\hline Gestion des extrants & - Mise en place du tri sélectif des déchets. & \\
\hline
\end{tabular}




\begin{tabular}{|c|c|c|}
\hline $\begin{array}{l}\text { Gestion de la qualité } \\
\text { de l'environnement }\end{array}$ & $\begin{array}{l}\text { - Aide du fonds d'aide et de soutien } \\
\text { aux initiatives étudiantes (FDSIE) : } \\
\text { financement du projet Vélocampus par } \\
\text { exemple (prêts et entretien de vélos). } \\
\text { - Mise en place d'un plan mobilité, de } \\
\text { circuits piétons et cyclables, réductions } \\
\text { des parkings voiture, remplacement des } \\
\text { véhicules par un parc propre au fur et à } \\
\text { mesure des déclassements de matériels, } \\
\text { vélos à assistance électrique de fonction } \\
\text { en cours d'achat. } \\
\text { - Délégation de la gestion des espaces verts } \\
\text { à Nantes Métropole qui assure une gestion } \\
\text { « verte ». } \\
\text { - Isolation thermique progressive des } \\
\text { anciens bâtiments. } \\
\text { - Inclusion des énergies renouvelables } \\
\text { dans toute nouvelle construction. }\end{array}$ & $\begin{array}{l}\text { - Signature d'un Plan mobilité } \\
\text { avec Nantes Métropole (qui offre } \\
\text { par exemple jusqu'à } 200 € \text { pour } \\
\text { l'achat d'un vélo électrique). } \\
\text { - L'association étudiante } \\
\text { «Bullivers» organise une } \\
\text { distribution de paniers de fruits } \\
\text { et légumes locaux en partenariat } \\
\text { avec l'ADDA (Association pour } \\
\text { le développement durable dans } \\
\text { l'alimentation). } \\
\text { - Redéfinition en } 2010 \text { de l'appel } \\
\text { d'offres pour la gestion des } \\
\text { espaces verts pour qu'il intègre } \\
\text { une gestion écologique. } \\
\text { - Audit énergétique en cours sur } \\
\text { l'ensemble des bâtiments. }\end{array}$ \\
\hline
\end{tabular}

